\title{
Exome sequencing reveals a de novo POLD1 mutation causing phenotypic variability in mandibular hypoplasia, deafness, progeroid features, and lipodystrophy syndrome (MDPL)
}

\author{
Sahar Elouej ${ }^{a}$, Ana Beleza-Meireles ${ }^{b}$, Richard Caswell ${ }^{c}$, Kevin Colclough $^{d}$, Sian Ellard ${ }^{d}$, \\ Jean Pierre Desvignes ${ }^{a}$, Christophe Béroud ${ }^{a, e}$, Nicolas Lévy $^{a, e}$, Shehla Mohammed ${ }^{b}$, \\ Annachiara De Sandre-Giovannoli ${ }^{a, e, *}$ \\ a Aix Marseille Univ, INSERM, GMGF, Marseille, France \\ b Department of Clinical Genetics, Guy's and St Thomas' NHS Foundation Trust, London, UK \\ ${ }^{c}$ Institute of Biomedical and Clinical Science, University of Exeter Medical School, Exeter, UK \\ d Department of Molecular Genetics, Royal Devon and Exeter NHS Foundation Trust, Exeter, UK \\ e Department of Medical Genetics, Molecular genetics Laboratory, La Timone Children's Hospital, 264 Rue Saint Pierre, 13005, Marseille, \\ France
}

\begin{abstract}
A B S T R A C T
Background. Mandibular hypoplasia, deafness, progeroid features, and lipodystrophy syndrome (MDPL) is an autosomal dominant systemic disorder characterized by prominent loss of subcutaneous fat, a characteristic facial appearance and metabolic abnormalities. This syndrome is caused by heterozygous de novo mutations in the POLD1 gene. To date, 19 patients with MDPL have been reported in the literature and among them 14 patients have been characterized at the molecular level. Twelve unrelated patients carried a recurrent in-frame deletion of a single codon (p.Ser605del) and two other patients carried a novel heterozygous mutation in exon 13 (p.Arg507Cys). Additionally and interestingly, germline mutations of the same gene have been involved in familial polyposis and colorectal cancer (CRC) predisposition.

Patients and Methods. We describe a male and a female patient with MDPL respectively affected with mild and severe phenotypes. Both of them showed mandibular hypoplasia, a beaked nose with bird-like facies, prominent eyes, a small mouth, growth retardation, muscle
\end{abstract}


and skin atrophy, but the female patient showed such a severe and early phenotype that a first working diagnosis of Hutchinson-Gilford Progeria was made. The exploration was performed by direct sequencing of POLD1 gene exon 15 in the male patient with a classical MDPL phenotype and by whole exome sequencing in the female patient and her unaffected parents.

Results. Exome sequencing identified in the latter patient a de novo heterozygous undescribed mutation in the POLD1 gene (NM_002691.3: c.3209T >A), predicted to cause the missense change p.Ile1070Asn in the ZnF2 (Zinc Finger 2) domain of the protein. This mutation was not reported in the 1000 Genome Project, dbSNP and Exome sequencing databases. Furthermore, the Isoleucine1070 residue of POLD1 is highly conserved among various species, suggesting that this substitution may cause a major impairment of POLD1 activity. For the second patient, affected with a typical MDPL phenotype, direct sequencing of POLD1 exon 15 revealed the recurrent in-frame deletion (c.1812_1814del, p.S605del).

Conclusion. Our work highlights that mutations in different POLD1 domains can lead to phenotypic variability, ranging from dominantly inherited cancer predisposition syndromes, to mild MDPL phenotypes without lifespan reduction, to very severe MDPL syndromes with major premature aging features. These results also suggest that POLD1 gene testing should be considered in patients presenting with severe progeroid features.

(c) 2017 Elsevier Inc. All rights reserved.

\section{Introduction}

Mandibular hypoplasia, deafness, progeroid features, and lipodystrophy syndrome (MDPL, OMIM\#615381), is a rare systemic disorder characterized by prominent loss of subcutaneous fat, a characteristic facial appearance and metabolic abnormalities. This disorder was clinically described for the first time by Shastry et al. in 2010 who reported seven subjects presenting with overlapping features with mandibuloacral dysplasia syndromes (MAD-A, due to recessive mutations in the LMNA gene, OMIM\#248370, and MAD-B, due to recessive ZMPSTE24 mutations, OMIM\#608612) such as mandibular hypoplasia, prominent eyes, stiff joints, beaked nose, and lipodystrophy, but with specific additional clinical features [1]. These included sensorineural hearing loss, hypogonadism and absent clavicular hypoplasia/acroosteolyses. MAD and MDPL belong to the group of diseases characterized by premature aging, which can be caused by inheritable nuclear envelope and/or DNA repair defects. Some of the major disorders included in this group are: Hutchinson-Gilford progeria syndrome (HGPS, OMIM\# 176670) caused by dominant mutations in the LMNA gene [2,3]; Nestor-Guillermo progeria syndrome (NGPS, OMIM\#614008), due to recessive mutations in the BANF1 gene [4]; Werner syndrome (WRN, OMIM\#277700), due to recessive mutations in the WRN gene [5]; Restrictive dermopathy (RD, OMIM\#275210), mainly due to recessive null mutations in the ZMPSTE24 gene [6,7]; Cockayne syndrome type A and B (CSA, OMIM\#216400; CSB, OMIM\#133540), due to mutations in ERCC8 and ERCC6 respectively [8,9] and Marfanprogeroid syndrome (MFLS, OMIM\# 616914) due to dominant mutations in FBN1 gene. Due to the clinical similarities among these syndromes and possible atypical phenotypes, clinical diagnosis can be quite a challenge, which can be solved in most cases through molecular exploration.

MDPL syndrome is an autosomal dominant disorder caused by mutations in the POLD1 gene encoding human polymerase delta 1 (POLD1), also known as the p125 subunit, which provides the essential catalytic and proofreading activities of DNA polymerase $\delta$ (Pol $\delta)[10,11]$. DNA polymerase $\delta$ is one of the three main enzymes responsible for DNA replication $[12,13]$ but it is also involved in multiple DNA repair mechanisms, including nucleotide excision repair, double strand break repair, base excision repair, and mismatch repair [14]. The catalytic subunit encoded by POLD1 is responsible for the synthesis of DNA lagging-strand by the combined action of two activities: 5 ' - to 3 '-polymerase activity and $3^{\prime}$ - to $5^{\prime}$-exonuclease or proofreading activity [15]. POLD1 is composed of 1107 amino-acids and includes several distinct functional domains: a putative nuclear localization signal (amino-acid 4 to amino-acid 19), an exonuclease domain (amino-acid 306 to amino-acid 519), a polymerase active site (amino-acid 581 to amino-acid 910) and a ZnF domain (aminoacid 1012 to amino-acid 1083) which contains two conserved cysteine-rich metal-binding motifs (CysA and CysB) $[15,16]$.

To date, 19 subjects with MDPL have been reported in the literature, whose phenotypes are similar and rather mild, without shortened lifespans. To the best of our knowledge, only two mutations in the POLD1 gene have been identified to date. The first is a recurrent de novo in frame deletion of a single codon (c.1812_1814delCTC, p.Ser605del) identified in 12 unrelated patients $[11,17,18]$ with confirmed pathogenicity, related to functional studies. The second is a de novo missense mutation (c.1572C > T, p.Arg507Cys) identified in two patients and first reported in a 48 years old woman [18,19]. These genetic changes respectively affect the polymerase domain and the C-terminal part of the proofreading (exonuclease) domain of the polymerase. Additionally, a very recent paper [20] reports a novel inherited POLD1 missense mutation in two related individuals (mother and daughter): c.3199 G>A; p.E1067K which was classified as likely pathogenic. The phenotype of these patients was partly different and milder compared to previously reported patients (please see the discussion section on phenotypic comparisons of POLD1 mutated patients). Interestingly, this is the first identified variant lying within the ZNF2 domain of POLD1. Finally, germline mutations in the exonuclease domains of POLD1 have been recently associated with familial polyposis and colorectal cancer predisposition $[10,21]$. 
In this study, we report the clinical and genetic investigation of two additional patients with variably severe MDPL syndromes, using either direct search of the POLD1 recurrent mutation by Sanger sequencing or whole exome sequencing (WES).

\section{Methods}

\subsection{Patients}

Two patients with MDPL were included in this study. They were admitted to the Guy's and St. Thomas' NHS Foundation Trust Hospital in London. The parents of each patient gave informed and written consents for the studies performed as well as for picture publication, including uncovered faces. The explorations were performed first in a diagnostic setting, then in a research setting, complying with the ethical guidelines of the institutions involved and the declaration of Helsinki. After written informed consent was obtained from the family members, peripheral blood samples from all of the participants were drawn, and genomic DNA was extracted following standard procedures. No cell lines could be established for functional studies in these two patients.

\subsection{Whole-Exome Sequencing}

Whole-exome sequencing was performed on patient 1 and her unaffected parents. Briefly, exome was captured from genomic DNA using Agilent SureSelect Clinical Research Exome kit and then sequenced on an Illumina HiSeq 4000 sequencer using 70-bp paired-end reads. We used BWA [22] to align sequence reads to the hg19 reference genome and GATK [23] to call SNVs and indels. Control quality showed that $88 \%$ of targeted bases were covered at $>20 x$.

\subsection{Whole-Exome Data Analysis}

The results were analyzed using the in-house VarAft software version 1.6, which is freely available online (http://varaft.eu/ index.php). Through exome analysis, both recessive and dominant models of inheritance have been performed (Table 2). We prioritized rare functional variants (missense, nonsense, splice site variants, and indels) that were heterozygous de novo mutations, homozygous, or compound heterozygous in the proband and excluded variants with a Minor Allele Frequency (MAF) >0.01 in dbSNP137, and 138, in the Exome Variant Server (http://evs.gs.washington.edu/EVS/), 1000 Genomes Project (http://www.1000genomes.org/), or Exome Aggregation Consortium database (ExAC), Cambridge, MA (http://exac.broadinstitute. org). A number of online tools were used to predict the functional impact and pathogenicity of the POLD1 variants such as MutationTaster (http://www.mutationtaster.org/), PredictProtein (https://www.predictprotein.org/), PolyPhen (http://genetics.bwh. harvard.edu/pph2/), Combined Annotation Dependent Depletion (CADD) (http://cadd.gs.washington.edu/), SIFT (http://sift.bii.astar.edu.sg/) and UMD predictor (http://umd-predictor.eu/).

\subsection{Sanger Sequencing Confirmation}

The Primer3 online tool (http://bioinfo.ut.ee/primer3-0.4.0/) was used to design the specific primers used for PCR amplification and direct sequencing of the surrounding region of each variant. Primers used to amplify the mutant sequence were POLD1-Ex15-F (5'-GCTCCCAGCCAATGAATG-3'), POLD1-Ex15-R (5'-CTACTATCTCTGGGCCTGTGC-3'), POLD1Ex26-F (5'-TCCCCAGGTATCCCATCTGA-3'), and POLD1Ex26-R (5'-TTGGAGTCAGAGGTTGGGTC-3'). PCR products were examined by agarose gel electrophoresis and subjected to Sanger sequencing. Exome results and the mutation segregation pattern were confirmed by Sanger sequencing.

\section{Results}

\subsection{Patients' Clinical Features}

Both patients were sporadic, isolated cases with no known consanguinity among parents. Both showed mandibular hypoplasia, a beaked nose with bird-like facies, prominent eyes, small mouth, growth retardation, limb muscle and skin atrophy (Table 1), but those features were much more marked in patient 1 (female patient, Fig. 1) compared to patient 2 (male patient, Fig. 2).

Patient 1 was one of three children born to unrelated parents. At age 18 months, as seen in the picture provided (Fig. 1a), the patient did not show any apparent clinical signs. At the age of 7-8 years, she presented with growth retardation, mid face hypoplasia with dental crowding, and low set ears. The clinical diagnosis was then subsequently revised to Hutchinson-Gilford Progeria but no testing was available at the time. She was first seen by us at the age of 23 years (Fig. 1b) having been under the care of the cardiac and respiratory teams with musculoskeletal problems and recurrent respiratory chest infections. She had been given a clinical diagnosis of Acrogeria/ Ehlers-Danlos syndrome type 4 originally but collagen studies were inconclusive. Routine investigations for failure to thrive, including thyroid and pituitary functions were negative and there was no effect from a trial of recombinant growth hormone. She was then seen at age of 24 years where she presented with extreme generalized amyotrophy and subcutaneous lipoatrophy (Fig. 1c,d). At age 24 years her height and weight were $1.20 \mathrm{~m}(<\mathrm{P} 1)$ and $12.6 \mathrm{~kg}(<\mathrm{P} 3)$, respectively and the body mass index was $8.75 \mathrm{~kg} / \mathrm{m}^{2}$. She had quite prominent eyes with a small jaw (Fig. 1e,f). She required extensive dental surgery because of crowded teeth (Fig. 1b,d). She also required treatment for exposure keratopathy. She had no secondary sex characteristics development and had primary amenorrhoea. Moreover, her skin was quite thin and sclerodermatous with prominent veins and lack of subcutaneous fat. (Fig. 1f,g,h); swelling of interphalangeal joints could be observed in the hands. She had stiffness and limited movements in elbows, knees and hips. She suffered from a major scoliosis, leading to several surgical interventions (Fig. 1i) as well as from severe and recurrent respiratory tract infections. Her clinical picture evolved towards a restrictive dermopathy phenotype and bulbar dysphagia necessitating a percutaneous endoscopic gastrostomy, until death occurred at 25 years of age, following a severe pulmonary infection. Of note, this patient did not present with hearing loss and showed entirely normal psychomotor development. The oral glucose intolerance test for patient 1 indicates an impaired glucose tolerance or a prediabetes 
Table 1-Comparison of the phenotypic characteristics of MDPL patients with pathogenic mutations in the POLD1 gene (literature and this study).

$\begin{array}{ll}\text { Present study } & \\ \text { Patient } 1 \quad \text { Patient } 2 & \end{array}$

Ajluni

Lessel et al., et al., 2015 (ranges

Reinier

Pelosini

Weedon 2017 of reported values)

et al., et al., et al., (ranges of 20152014

2013 reported

(ranges of values, reported POLD1 values)

mutated)

\begin{tabular}{|c|c|c|c|c|c|c|c|}
\hline Age (y) at referral & 24 & 6 & $14-34$ & $10-62$ & 41 & 48 & $19-37$ \\
\hline Sex & $\mathrm{F}$ & $\mathrm{M}$ & $2 \mathrm{~F}$ & $3 \mathrm{M} / 5 \mathrm{~F}$ & $\mathrm{~F}$ & $\mathrm{~F}$ & $3 \mathrm{M} / 1 \mathrm{~F}$ \\
\hline Age at onset (y) & 7 & 5 & NR & 10-20 (1 NR) & 3 & 5 & $2.5-4$ (2 NR) \\
\hline Age of death (y) & $\begin{array}{l}25 \\
\text { (respiratory } \\
\text { failure) }\end{array}$ & NA & NR & 34, ovarian cancer $(1 / 8)$ & NR & NR & NR \\
\hline Ethnicity & British & British & $\begin{array}{l}\text { White- } \\
\text { Hispanic }\end{array}$ & $\begin{array}{l}3 \text { French; } 2 \text { US, mixed } \\
\text { European; } 1 \text { Swedish; } 1 \\
\text { Hungarian, } 1 \text { Colombian }\end{array}$ & Italian & Italian & $\begin{array}{l}2 \text { British; } 1 \\
\text { Italian; } 1 \\
\text { Indian }\end{array}$ \\
\hline Birth weight (kg) & 2.7 & 3.18 & NR & $2.4-3.30$ (4 NR) & NA & 3.6 & $3-4.2$ \\
\hline Height (m) (Z-score) & $1.2(-6.6)$ & $1.114(-0.9)$ & $\begin{array}{l}163-171 \\
(1.19 ; 0.38)\end{array}$ & $123-162(-0.9 ;-3.7)$ & $1.45(-2.8)$ & $1.62(-0.27)$ & $\begin{array}{l}1.60-1.91 \\
(-0.50 ; 1.99)\end{array}$ \\
\hline Weight (kg) & 12.6 & 16.24 & $50.4-53.0$ & $21-66$ & 33 & 47.7 & $41.3-69.7$ \\
\hline $\begin{array}{l}\text { BMI }\left(\mathrm{kg} / \mathrm{m}^{2}\right) \\
\text { Clinical characteristics }\end{array}$ & 8.8 & 13.09 & $18.1-19.0$ & $13.8-26.8$ & 15.7 & 18.2 & $14.6-19.1$ \\
\hline Lipodystrophy & + & + & + & 8/8 Yes & + & + & 4 Yes \\
\hline Mandibular hypoplasia & + & + & - & $7 / 8$ Yes & + & + & 4 Yes \\
\hline pinched facies & + & + & - & $8 / 8$ & + & + & 4 yes \\
\hline Beaked nose & + & + & - & $8 / 8$ & + & + & 4 yes \\
\hline High-pitched voice & - & - & - & 5/8 Yes & + & NR & 4/4 Yes \\
\hline $\begin{array}{l}\text { Graying or loss } \\
\text { of hair }\end{array}$ & - & + & - & 3/8 Yes & - & - & $0 / 4$ \\
\hline Joint contractures & + & + & + & 1/8 Yes & - & + & 2/4 Yes \\
\hline Telangiectasia & + & - & - & 5/8 Yes & + & - & 3/4 Yes \\
\hline Acanthosis & - & + & - & $0 / 8$ & - & - & $0 / 4$ \\
\hline Crowded teeth & + & + & - & $5 / 8$ Yes & + & + & 4/4 Yes \\
\hline $\begin{array}{l}\text { Deafness (age of } \\
\text { onset, y) }\end{array}$ & - & $+(4)$ & - & 5/8 Yes (11-14) & 10 & 25 & $\begin{array}{l}\text { 4/4 Yes } \\
(7-33)\end{array}$ \\
\hline $\begin{array}{l}\text { Lack of breast } \\
\text { development }\end{array}$ & + & NA & - & NR & + & + & $\begin{array}{l}1 / 4 \text { Yes } \\
\text { (3 NA) }\end{array}$ \\
\hline Short stature & + & + & - & 7/8 Yes & + & - & $0 / 4$ \\
\hline Corneal keratosis & + & - & - & $0 / 8$ & - & - & 4/4 Normal \\
\hline Kyphosis/scoliosis & + & - & - & NR & - & + & 3/4 Yes \\
\hline Osteoporosis & + & - & & 2/8 Yes (1 NR) & - & - & 3/4 Yes \\
\hline Hypogonadism & + & $\begin{array}{l}\text { Right } \\
\text { chryptorchidism } \\
\text { and phymosis } \\
\text { under investigation }\end{array}$ & $\begin{array}{l}+ \\
\text { Polycystic } \\
\text { ovary } \\
\text { syndrome }\end{array}$ & $\begin{array}{l}\text { 1/8 Yes } \\
\text { (Cryptorchidism) }\end{array}$ & + & - & 3/4 Yes \\
\hline Cognitive function & Normal & Normal & - & $\begin{array}{l}\text { 6/8 Normal; } \\
1 \text { Depression, anxiety; } \\
1 \mathrm{NR}\end{array}$ & - & - & 4/4 Normal \\
\hline $\begin{array}{l}\text { Respiratory tract } \\
\text { infections } \\
\text { Metabolic profile }\end{array}$ & + & - & - & NR & NR & NR & NR \\
\hline $\begin{array}{l}\text { Hepatic steatosis } \\
\text { (years) }\end{array}$ & - & - & + & $1 / 8(6 \mathrm{NR})$ & (22) & + & $\begin{array}{l}2 / 4 \text { Yes } \\
\text { (1 NR) }\end{array}$ \\
\hline Hepatomegaly & - & - & - & 3/8 Normal (5 NR) & + & + & $\begin{array}{l}1 / 4 \text { Yes } \\
\text { (1 NR) }\end{array}$ \\
\hline Hypertriglyceridemia & - & - & + & 5/8 (2 NR) & + & 25 & 2/4 Yes \\
\hline $\begin{array}{l}\text { Diabetes (age at } \\
\text { diagnosis) }\end{array}$ & $?$ & - & + & 2/8 Yes (28-43) & 30 & - & $\begin{array}{l}3 / 4 \text { Yes } \\
(14-37)\end{array}$ \\
\hline $\begin{array}{l}\text { Triglycerides } \\
\text { (mmol/l) }\end{array}$ & NA & NA & $5.87-7.92$ & $2.15-2.62$ (2NR) & 3.47 & NR & $1.48-2.6$ \\
\hline $\begin{array}{l}\text { Total Cholesterol } \\
\text { (mmol/l) }\end{array}$ & NA & 2.9 & NR & $2.99-5.87$ (2NR) & 5.53 & NR & $4.2-5.4$ \\
\hline HDL-C (mg/dl) & NA & 49 & NR & 25-47 (4 NR) & 47 & NR & $\begin{array}{l}16-35 \\
(1 \mathrm{NA})\end{array}$ \\
\hline
\end{tabular}




$\begin{array}{lllll}\text { Present study } & \text { Ajluni } & \text { Lessel et al., } & \text { Reinier } & \text { Pelosini } \\ \text { Patient 1 Patient 2 } & \text { et al., } & \text { 2015 (ranges } & \text { et., } & \text { et al., } \\ & 2017 \quad \text { of reported values) } & 2015 & 2014 \\ & \text { (ranges of } & & \\ & \text { reported } & & \\ & \text { values, } & & \\ & \text { POLD1 } & & \\ & \text { mutated) } & & \\ & & \end{array}$

Weedon et al., 2013 (ranges of reported values)

\begin{tabular}{|c|c|c|c|c|c|c|c|}
\hline Leptin (ng/ml) & NA & NA & $9.0-11.5$ & NR & NR & 5.4 & $\begin{array}{l}4.5-8.22 \\
(1 \mathrm{NA})\end{array}$ \\
\hline $\begin{array}{l}\text { Fasting insulin } \\
(\mathrm{pmol} / \mathrm{l})\end{array}$ & NA & 357 & NR & NR & 190 & 243 & $\begin{array}{l}169-235 \\
(1 \mathrm{NR})\end{array}$ \\
\hline $\begin{array}{l}\text { Insulin Like Growth Factor } \\
\text { (nmol/l) (Normal ranges) }\end{array}$ & 11. $3(13-50)$ & 25.7 & NR & NR & NR & NR & NR \\
\hline $\begin{array}{l}\text { OGTT fasting glucose } \\
(\mathrm{mmol} / \mathrm{l})\end{array}$ & 8.4 & $<5.6$ & NR & $4.22-5.21$ (1 NR) & NR & 5.28 & $4.2-7.7$ \\
\hline $\begin{array}{l}\text { OGTT } 2 \mathrm{~h} \text { glucose } \\
(\mathrm{mmol} / \mathrm{l})\end{array}$ & NA & NA & $\mathrm{NR}$ & NR & NR & 10.2 & $8.9-16.4$ \\
\hline $\mathrm{HbA1c}(\mathrm{mmol} / \mathrm{mol})$ & NA & 31.1 & $90.2-91.3$ & 27.9-37.7 (4 NR) & NR & 44 & $\begin{array}{l}36-49 \\
(1 \mathrm{NR})\end{array}$ \\
\hline Abnormal LFTs ever & - & NA & NR & NR & NR & + & 3/4 Yes \\
\hline $\begin{array}{l}\text { ALT }(\mathrm{u} / \mathrm{L}) \text { (Normal } \\
\text { ranges) }\end{array}$ & $11(4-45)$ & NA & $51-97$ & 25-44 (4 NR) & 53 & NR & $32-172$ \\
\hline $\begin{array}{l}\text { ALP (U/L) (Normal } \\
\text { ranges) }\end{array}$ & $96(35-129)$ & NA & $\mathrm{NR}$ & $\mathrm{NR}$ & 47 & $\mathrm{NR}$ & $\begin{array}{l}66-103 \\
(1 \mathrm{NR})\end{array}$ \\
\hline $\begin{array}{l}\text { BIL ( } \mu \mathrm{mol} / \mathrm{L}) \text { (Normal } \\
\text { ranges) }\end{array}$ & $6(0-21)$ & NA & NR & $5.1-13.6$ (4 NR) & 3.4 & $\mathrm{NR}$ & $5.6-12$ \\
\hline $\operatorname{ALB}(g / L)$ & 96 & NA & $\mathrm{NR}$ & NR & NR & NR & $43-53.7$ \\
\hline FSH (UI/l) & 8 & NA & NR & NR & 4.07 & NR & NR \\
\hline LH (UI/l) & 4.9 & NA & NR & NR & 2.30 & NR & NR \\
\hline $\begin{array}{l}\text { Hypothyroidism } \\
\text { (age at diagnosis) }\end{array}$ & - & - & NR & $1 / 8(14)$ & - & - & - \\
\hline $\begin{array}{l}\text { Prolactin (ng/ml) } \\
\text { (Normal ranges) }\end{array}$ & $70(102-496)$ & NA & NR & NR & 43.85 & NR & NR \\
\hline \multicolumn{8}{|l|}{ Fat distribution (DEXA) } \\
\hline $\begin{array}{l}\text { Whole-body fat } \\
\text { (\% body mass) }\end{array}$ & NA & NA & NR & NR & NR & 24.5 & $14.0-22.6$ \\
\hline $\begin{array}{l}\text { Truncal fat (\% } \\
\text { regional mass) }\end{array}$ & NA & NA & $40.4-58.3$ & NR & NR & 29.9 & $\begin{array}{l}19.1-22.6 \\
(1 \mathrm{NR})\end{array}$ \\
\hline $\begin{array}{l}\text { Arm fat (\% regional } \\
\text { mass) }\end{array}$ & NA & NA & NR & NR & NR & NR & $\begin{array}{l}4.0-20.4 \\
(1 \mathrm{NR})\end{array}$ \\
\hline $\begin{array}{l}\text { Leg fat (\% regional } \\
\text { mass) }\end{array}$ & NA & NA & $11.9-23.2$ & NR & NR & NR & $\begin{array}{l}4.8-13.9 \\
(1 \mathrm{NR})\end{array}$ \\
\hline $\begin{array}{l}\text { Truncal fat (\%)/leg } \\
\text { fat (\%) }\end{array}$ & NA & NA & $1.43-2.01$ & NR & NR & 2 & $\begin{array}{l}1.71-22.1 \\
(1 \mathrm{NR})\end{array}$ \\
\hline POLD1 mutation & p.Ile1070Asn & p.Ser605del & p.E1067K & $\begin{array}{l}\text { 7/8 p.Ser605del; } 1 / 8 \\
\text { p.Arg507Cys }\end{array}$ & p.Ser605del & p.Arg507Cys & $\begin{array}{l}4 / 4 \\
\text { p.Ser605del }\end{array}$ \\
\hline
\end{tabular}

NR: not reported; NA: not available; +: Yes; -: No; LFTs = abnormal liver function tests;?: not confirmed.

status (Table 1). Unfortunately, it was not possible to assess whether she was affected with diabetes or to get further biochemical measurements related to dyslipidemia since she passed away before her disease could be diagnosed.

The second patient was a 6-year-old boy, born to healthy non consanguineous parents, following an uneventful pregnancy. He was born in good condition and with normal birth weight. From the age of 2 years short stature was observed and he started to have poor weight gain by the age of 5 years. He was diagnosed at age four with sensory neural low frequency hearing loss for which he wears bilateral hearing aids. He was referred for genetics assessment at age 7 years because of failure to thrive and extremely mild acanthosis nigricans on the sides of his neck and also in the axillas. At physical examination he presented with mild fat loss in his lower limbs, prominent eyes, pinched nose, small chin and thin lips (Fig. 2). He had proportionate short stature and low weight for age, height $1.114 \mathrm{~m}$, weight $16.2 \mathrm{~kg}$ and the body mass index was $13.09 \mathrm{~kg} / \mathrm{m}^{2}$ (<P2 for height and weight, OFC on the 25th-50th centile). He also showed joint restriction, particularly in shoulder extension (Fig. 2d). Further, genitalia examination and further explorations showed phimosis and right cryptorchidism. He had a normal psychomotor development, normal speech and language development and unremarkable skeletal survey. A recent change in hair texture was noted together with the appearance of white hair. Abdominal 

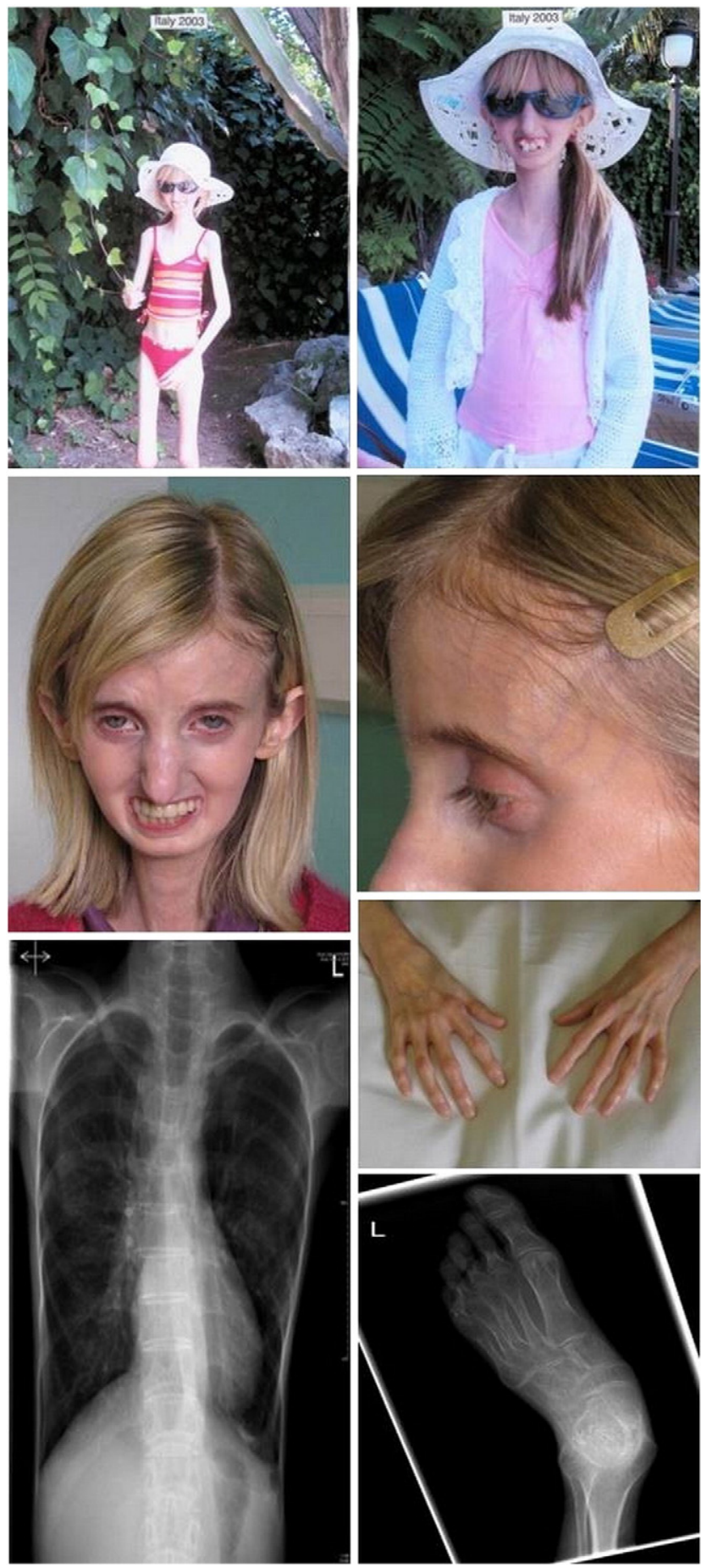

Fig. 1 - Clinical characteristics of patient 1 affected with MDPL syndrome. (a): Patient 1 at age 18 months with normal appearance; (b-h): pictures of patient 1 at different ages from 13 (b) to 23 years $(f, g)$, showing prominent eyes, beaked nose, a small jaw with crowded teeth, major lipodystrophy with thin skin and prominent superficial vessels; in panels e and $f$ dental crowding had been surgically corrected (i-l): radiographs showing the scoliosis of the mid thoracic spine and other skeletal findings, including arachnodactyly, severe osteoporosis with thin cortices and very slender diaphysis. ultrasound did not show any pathological features and no increased intra-abdominal adipose tissue. X-rays of the skeleton showed a delayed bone age. Routine biochemical test revealed a normal oral glucose tolerance test and did not highlight any lipid profile dysregulation (Table 1).

Table 1 summarizes the clinical information concerning the two patients we report and all the patients previously reported in the literature. In particular, in order to better compare the linear growth of all the patients carrying POLD1 mutations leading to syndromic phenotypes, we computed their growth Z-scores on the basis of available information.

No chromosomal imbalance was detected by CGH array for both patients.

\subsection{Molecular Genetics Explorations}

Since patient 1 was first diagnosed as having a progeroid syndrome similar to HGPS or NGPS, due to strikingly overlapping clinical features, the coding exons and intronic boundaries of the LMNA, ZMPSTE24 and BANF1 genes were first screened by direct Sanger sequencing. No causative mutation was found in these genes. Therefore, samples from patient 1 and her healthy parents were subjected to whole exome sequencing in a research context, in order to search for the genetic defect underlying patient 1's disease. A summary of the bio-informatic workflow of this study is presented in Table 2. During our analysis, we have focused on missense, nonsense, splice-site, and frameshift variants upon all modes of inheritance. After sequential filtering as shown in Table 2, and keeping variants predicted as pathogenic, no homozygous variant nor compound heterozygous variants were identified in our patient, while only one heterozygous de novo variant was observed in the POLD1 gene. Moreover, important progeria and progeria-linked genes (LMNA, ZMPSTE24, BANF1, WRN, ERCC8, ERCC6, FBN1 and B4GALT7) were well covered and did not show any potentially pathogenic variant. Likewise, no mutations in genes associated to inherited lipodystrophies (i.e., AGPAT2, PPARG, BSCL2, LMNB2, CAV1, AKT2, FBN1, PIK3R1 etc.) were observed. We thus identified an unreported heterozygous c.3209 T>A (p.Ile1070Asn) missense mutation in the 26th coding exon of the POLD1 gene (NM_002691.3), which was absent from parental DNA on exome sequencing results (Fig. 3a). Bidirectional Sanger sequencing confirmed the de novo appearance of the c.3209 $\mathrm{T}>\mathrm{A}$ mutation in the patient (Fig. 3b). The c.3209 T>A variant was absent from dbSNP138, the 1000 Genomes Project, ExAC databases and the Exome Variant Server. The ExAC database reports the constraint metric for missense variants in POLD1 as 2.79 (i.e. the number of observed variants is 2.79 standard deviations below the mean value expected for the null hypothesis; [24]). Missense variants have been reported at 293/1107 residues of POLD1 (26.5\%), at an average allele frequency of $6.44 \times 10-4$ (Fig. 4). However, within the CysB region (residues 1058-1076), although missense variants occur at a similar proportion of residues (5/19) the average variant allele frequency of $5.32 \times 10-5$ is $~ 12$-fold lower than that over the entire coding region. This suggests that while there has been selective pressure against coding variation in POLD1 as a whole, this is greater within the CysB region. Specifically, no ExAC variants 

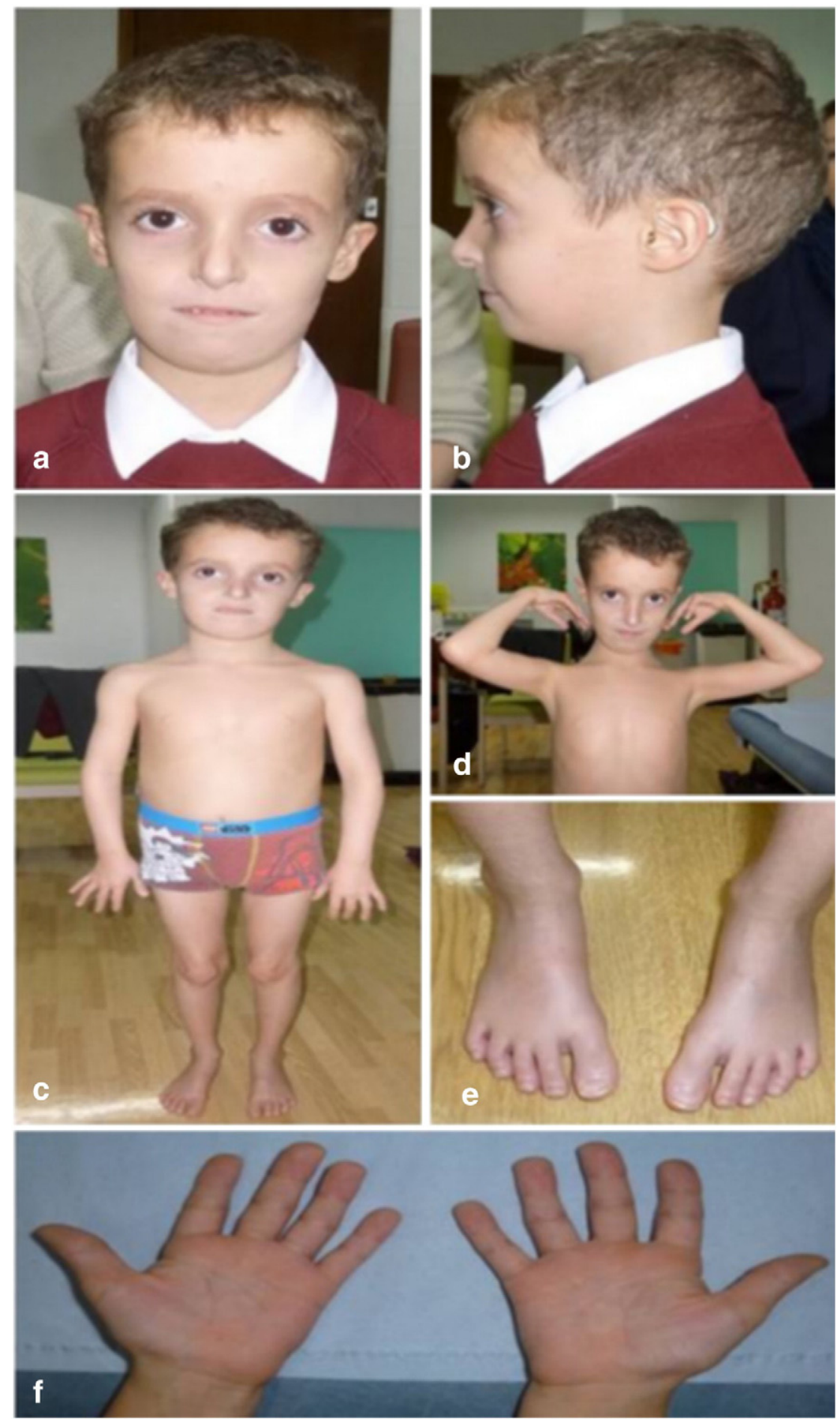

Fig. 2 - Clinical features of patient 2 affected with MDPL syndrome at 6 years of age. (a-c): mild lipodystrophy, beaked nose, prominent eyes, mandibular hypoplasia and hearing aids can be observed, together with reduced shoulder extension (d). (e-f): No dermatological or skeletal abnormality was observed in patient 2.

have been reported at Ile1070, the preceding residue Val1069, or any of the conserved cysteine residues $(1058,1061,1071$, and 1076), indicating that these residues are likely to be particularly important for normal function of this region.

Furthermore, this mutation was predicted as deleterious according to prediction databases such as SIFT, UMD predictor and Mutation Taster. Moreover, the CADD C-score of the variant was 25.7 , clearly predicting that the p.Ile1070Asn as deleterious (deleterious variants show scores $>15$ ). This variant lies outside the known enzymatic domains (C306C910) but within a region termed ZnF2 domain, also called CysB motif [16,25], located near the C-terminus (Fig. 5b). Although previously thought to be a zinc-binding motif, this region has recently been reported to form an iron-sulfur 
cluster of the $4 \mathrm{Fe}-4 \mathrm{~S}$ type and to be crucial for the assembly of the active DNA polymerase $\delta$ complex [25]. To discuss the significance of these sequence alterations, we compared the local amino-acid sequences among several organisms (Fig. 5c). Inspection of the genomic context around the variant shows that residues within the CysB motif (Cys1058-Cys1076) are strongly conserved, although isoleucine 1070 itself is only present in mammals whereas leucine is observed at this position in other vertebrates (Fig. 5c).

The crystal structure for the extreme C-terminus of POLD1, including the CysB region, has not yet been solved. Furthermore, interrogation of protein structure databases did not yield any suitable templates for comparative modeling of the 4Fe-4S cluster within CysB. Therefore to evaluate the effect of the Ile1070Asn substitution, full-length sequences of wildtype and variant POLD1 were submitted to the PredictProtein server [26] for prediction of protein secondary structure and amino acid solvent accessibility. The analysis of results over the CysB region showed that the p.Ile1070Asn substitution was predicted to have a significant effect on both the local secondary structure and the solvent accessibility of amino acid side chains within this region, and in particular around residues Cys1071 and Cys1076 which form part of the ironsulfur cluster (Fig. 6).

On the basis of family history and clinical presentation, patient 2 was clinically diagnosed as having MDPL syndrome and the recurrent mutation located in exon 15 of the POLD1 gene was directly searched for. Sanger sequencing confirmed the presence of the heterozygous in-frame deletion mutation in the POLD1 gene (c.1812_1814del, p.S605del; data not shown), confirming the clinical diagnosis. Parental testing confirmed the de novo appearance of the deletion in the patient.

\section{Discussion}

Monogenic disorders characterized by premature aging are clinically heterogeneous with overlapping phenotypic presentations, making the clinical diagnosis quite a challenge, especially in cases with variable severity. Molecular genetics analysis is a key tool to confirm the clinical diagnosis and search for the causal genes in atypical forms. Through exome sequencing we could successfully characterize at the molecular level a patient (patient 1) who first had a working diagnosis of Hutchinson Gilford Progeria Syndrome, given the particularly severe clinical presentation. Whole-exome sequencing of patient 1 and her unaffected parents revealed a heterozygous de novo missense mutation c.3209 T>A (p.Ile1070Asn) in the POLD1 gene. Several elements made us retain this variant as the pathogenic variant causing the patient's clinical picture: no missense variants have been reported in the ExAC database at this position or at neighboring residues, suggesting that these nucleotides are under strong selective pressure. The p.Ile1070Asn is located within the CysB region, a highly-conserved motif across eukaryotic DNA polymerases [16,25]: isoleucine 1070 is present in mammals whereas leucine, a chemically similar amino-acid, is observed at this position in other vertebrates. Apparently, during the divergence of mammals from other vertebrates, leucine became substituted by isoleucine, but since the side chains of these amino acids have very similar physicochemical properties, this substitution was tolerated and has been retained in all subsequent speciation. A corollary of this observation is that substitution of isoleucine for asparagine at this position, as observed in patient 1 , is unlikely to be tolerated. Indeed, in silico structural analysis using the PredictProtein server shows that the p.Ile1070Asn substitution is predicted to alter the local secondary structure and solvent accessibility within the CysB region, with the likely effect that the Fe-S cluster is destabilized. Both secondary structure and amino acid accessibility probably play a key role in the correct folding of this region into the Fe-S cluster, and specifically the cysteine side chains should remain buried and in the correct orientation to bind iron. In this respect it is notable that not only is residue 1070 always the hydrophobic leucine or isoleucine, but that the preceding residue is also a highly conserved non-polar residue. The substitution of Ile1070 by asparagine, a polar residue, is likely to cause displacement of the protein backbone to favor solvation of the novel side chain, with consequences for both the local structure and positioning of the C1071 side chain. Taken together, it is very likely that the Ile1070Asn substitution will cause disruption of the Fe-S cluster, and, since this has been shown to be crucial for interaction with other subunits in the assembly of the active polymerase complex, impairment of polymerase function [25]. An earlier study also demonstrated that removal of the tandem zinc finger modules located at the

Table 2 - Variant filtering results of whole exome sequencing for patient 1 (The number of variations decreases from top to bottom by applying each described filter).

\begin{tabular}{|c|c|c|c|}
\hline \multirow[b]{2}{*}{ Filtering conditions/inheritance models } & \multicolumn{2}{|l|}{ Autosomal recessive } & \multirow{2}{*}{$\begin{array}{l}\text { Autosomal } \\
\text { dominant } \\
\text { de novo }\end{array}$} \\
\hline & $\begin{array}{l}\text { Homozygous in patient } 1 \text { and } \\
\text { heterozygous in parents }\end{array}$ & Heterozygous composite & \\
\hline Total number of variants & 3427 & 10,974 & 2150 \\
\hline Missense, nonsense, splice-site or frameshift variants & 458 & 1553 & 244 \\
\hline MAF $<1 \%$ in the 1000 Genome, ExAC, ESP & 0 & 143 & 32 \\
\hline CADD score $>10$ & 0 & 111 & 17 \\
\hline Prediction in silico (UMD, SIFT, MutationTaster etc.) & 0 & 36 & 8 \\
\hline Remove dbSNP138 registered variants & 0 & 6 & 2 \\
\hline IGV visualization & 0 & 6 & 1 \\
\hline Selected mutation & 0 & 0 & POLD1:p.Ile1070Asn \\
\hline
\end{tabular}




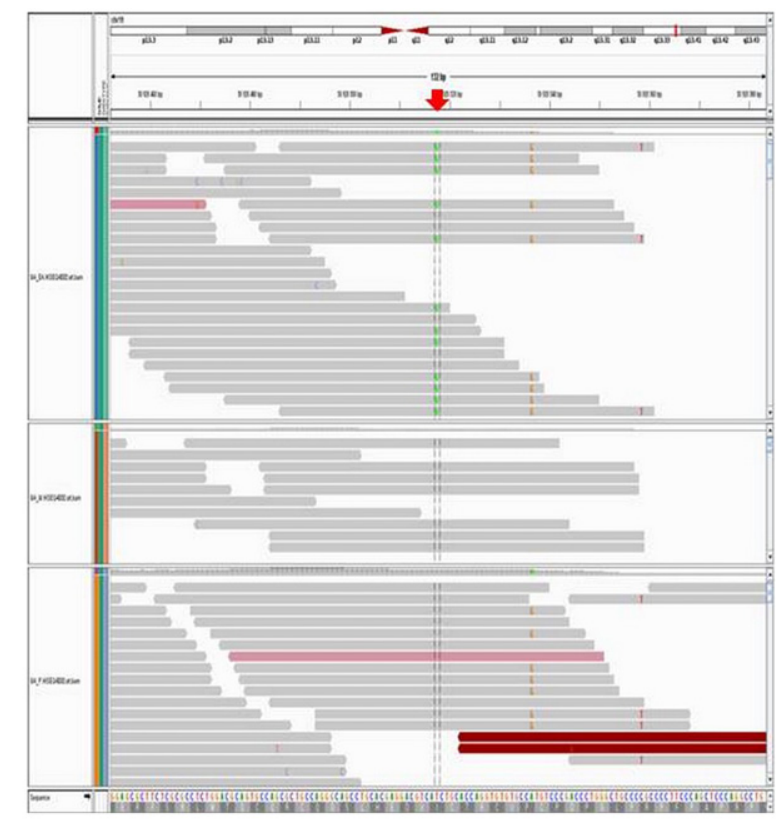

a
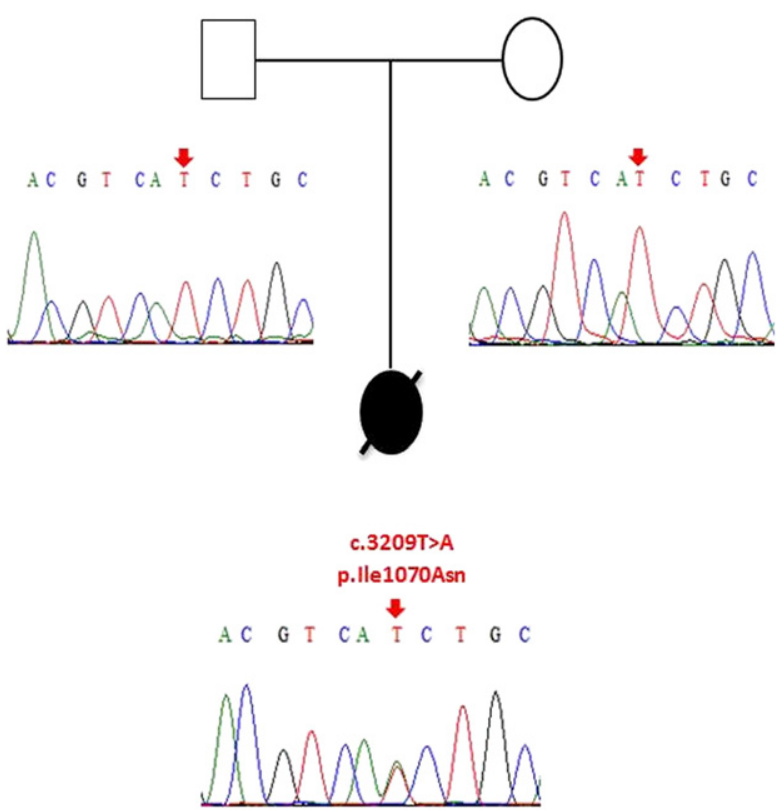

b

Fig. 3 - Whole exome results for patient 1.a: IGV browser visualization of whole exome sequencing results showing the heterozygous c.3209 T > A substitution in the POLD1 gene in patient 1 (upper panel), which is absent in her unaffected parents (mid and lower panels). b: The POLD1 c.3209 T > A p.lle1070Asn was validated by Sanger sequence analysis. Sanger sequencing of patient 1 and her parents confirm the de novo appearance of the of the p.Ile1070Asn mutation in the affected child.

C-terminus of Pol3 in yeast by targeted proteolysis, renders the Pol3 protein non-functional in vivo, and that the Cterminal zinc finger module $\mathrm{ZnF} 2$ is both necessary and sufficient for binding to the B-subunit of Pol3 in vivo and in vitro [16]. Taken together, it is highly likely that the p.Ile1070Asn variant leads to loss of polymerase $\delta$ activity,

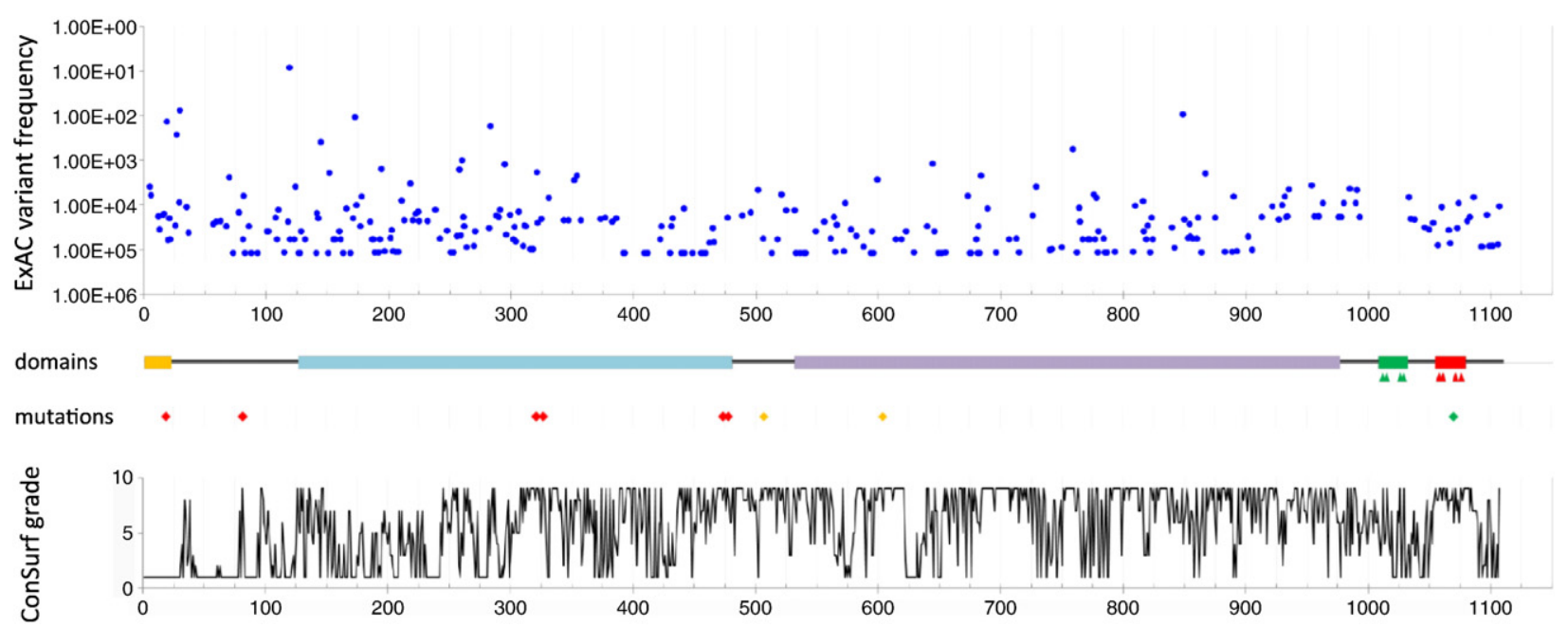

Fig. 4 - Variations and conservation in POLD1. Upper figure: ExAC missense variants in POLD1 (residues 1-1107) are plotted by position ( $x$-axis) against allele frequency (y-axis); missense variants occur at 293/1107 residues (26.5\%), at an average frequency of $6.44 \times 10-4$; data are not plotted for positions without an ExAC variant. Upper middle figure: functional domains in POLD1; orange: nuclear localization signal; blue: exonuclease ('proof-reading') domain; purple: polymerase domain; green: CysA motif; red: CysB motif; triangles below CysA/B motifs show positions of conserved cysteine residues. Lower middle figure: mutations in POLD1; red rhomboids show positions of HGMD mutations reported in colorectal adenoma, carcinoma and adenomatous polyposis; orange rhomboids show positions of HGMD mutations reported in MDPL (mandibular hypoplasia, deafness, progeroid features and lipodystrophy) syndrome; the green rhomboid shows the position of the novel variant p.I1070N. Lower figure: ConSurf grades for POLD1 1-1107, scored from 1 (minimum) to 9 (maximum). 


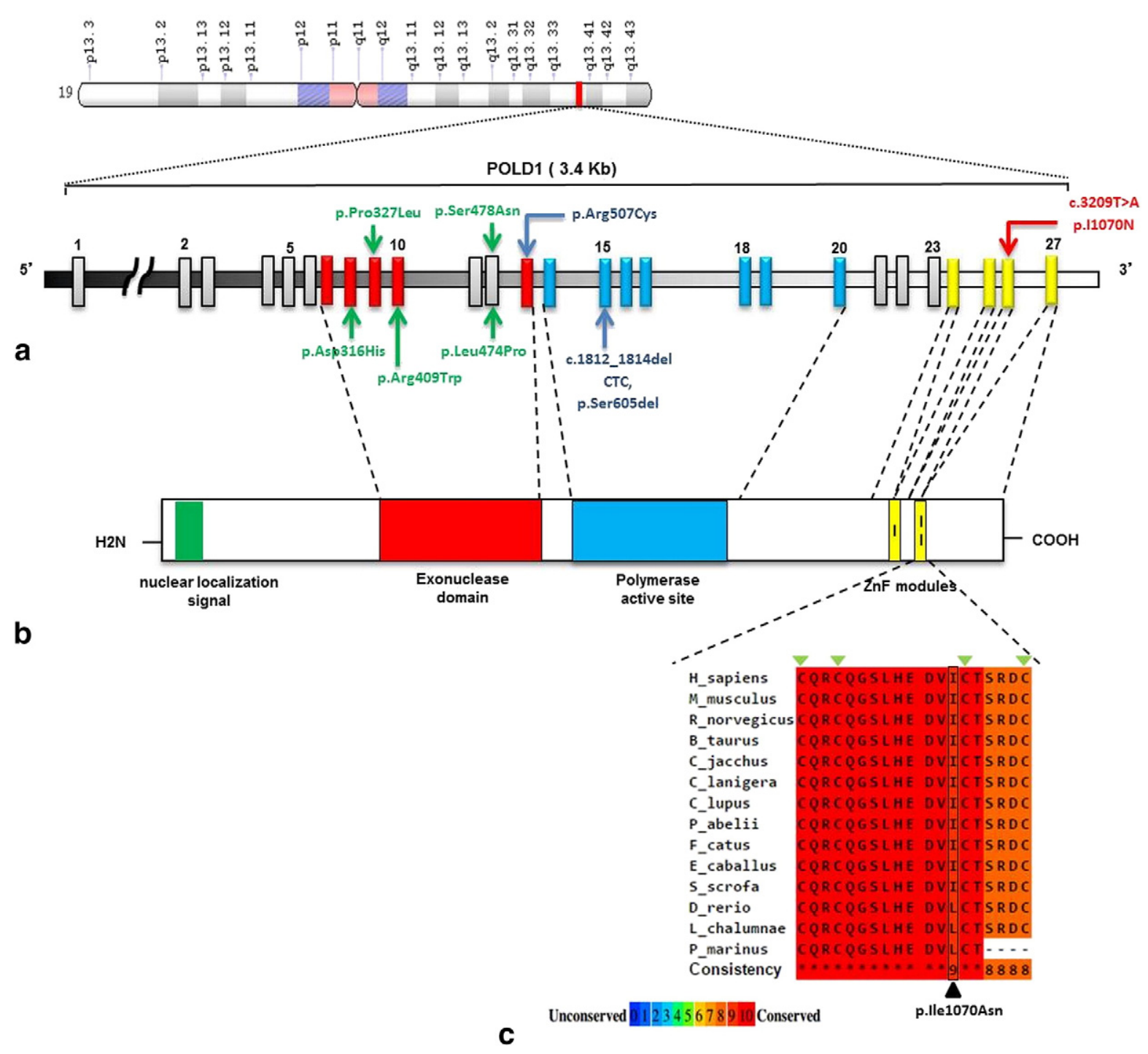

Fig. 5 - Genetic characterization of the POLD1 gene and its protein product. a: POLD1 is located on chromosome 19 at q13.3q13.4 and covers an approximately $3.4 \mathrm{~kb}$ long chromosomal region, containing 27 exons. Previous mutations in MDPL syndrome patients are indicated in blue and the new identified mutation in red. Germinal mutations reported in patients affected with colorectal adenoma are indicated in green. b: Schematic of the functional domains of POLD1 protein showing the relative locations of: nuclear localization signal in green; the exonuclease domain in red; the polymerase active site in blue and the ZnF modules (labeled I: CysA and II: CysB) in yellow. The exons encoding each domain are indicated in the corresponding color. c: Phylogenetic conservation of POLD1-CysB amino-acid sequence among different species. The position of the aminoacid substitution found in patient 1 is indicated by the dark arrow. The four invariant cysteine residues are indicated by the green arrows.

and a resulting severe phenotype sharing features with MDPL syndrome. Interestingly, a very recent study reported a mother and a daughter carrying a novel heterozygous variant in POLD1 exon 26 (c.3199G >A; p.E1067K), which is also highly conserved and which was predicted by the authors as likely pathogenic [20]. Glutamine 1067 lies within the ZNF2 domain, just as our mutant, thus supporting its pathogenic effect. For the first time, the authors used immunofluorescence staining to analyze the nuclear morphologies in liver sections of patients carrying a POLD1 mutation: interestingly, this analysis showed a high degree of nuclear atypia and disorganization, although at a lower extent than that observed in lipodystrophic patients carrying LMNA mutations. This observation interestingly supports possibly shared pathogenic mechanisms among POLD1 and LMNA-related lipodystrophic syndromes.

Unfortunately, it was not possible to conduct more in-depth cellular studies in patient 1 since she passed away before the identification of the mutation p.Ile1070Asn and no cell line had been established until then. But it will be certainly interesting to perform similar studies, on fibroblast cell lines or, if indicated, in liver biopsies, on other patients carrying POLD1 mutations as our patient 2 or previously published patients, both affected with MDPL or with mutations predisposing to colorectal cancer, to check whether abnormal nuclear morphology is a common 


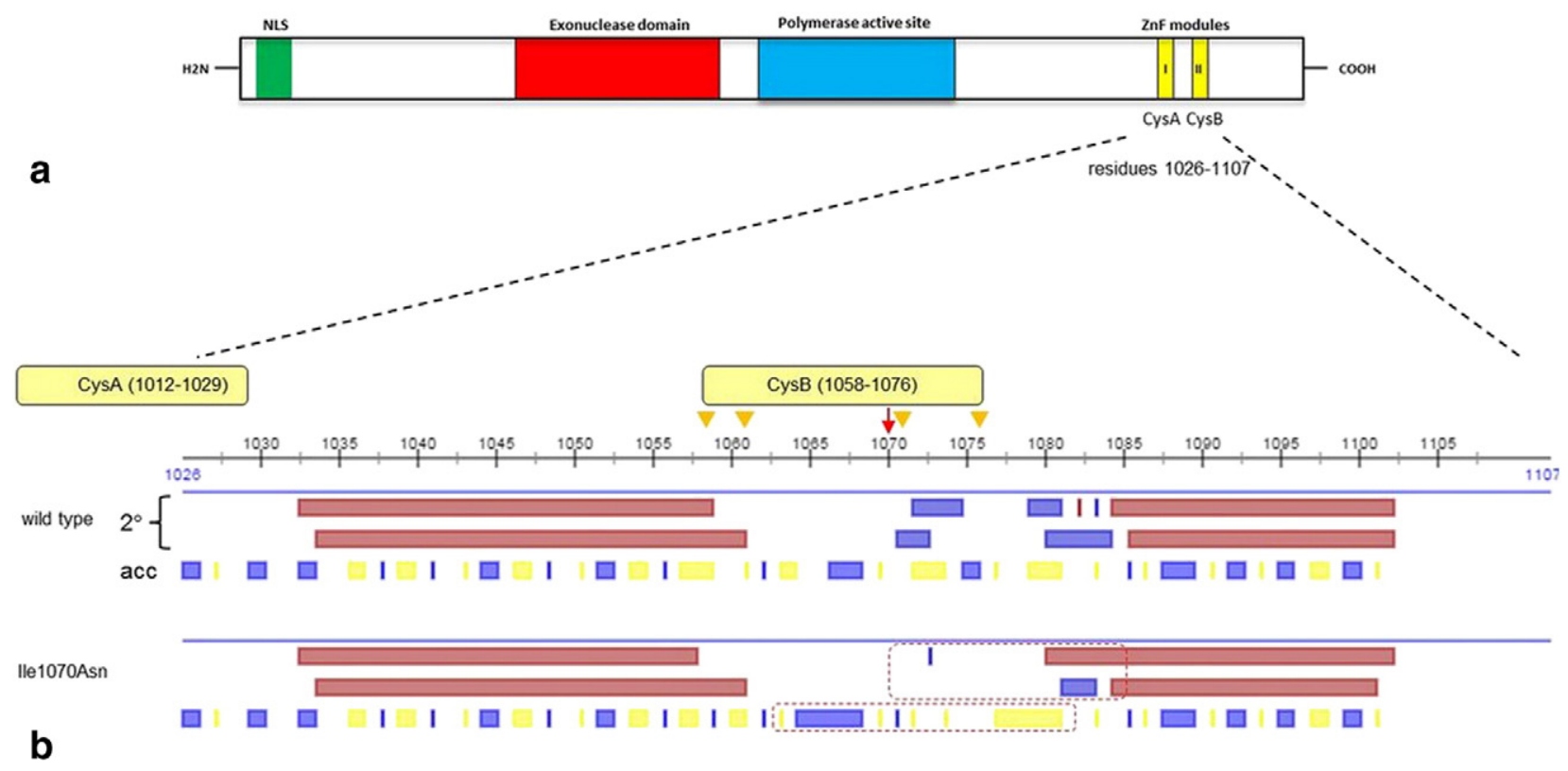

Fig. 6 - Analysis of POLD1 secondary structure using the PredictProtein server. a: Functional domains of POLD1, as shown in Fig. 5b. b: Secondary structure and solvent accessibility predictions from PredictProtein for wild-type and variant POLD1 (data shown for residues 1026-1107 only). The upper line shows a numbered scale; CysA (partial) and CysB regions are indicated by yellow boxes, and the position of residue 1070 is indicated by a red arrow; gold triangles represent positions of the four invariant cysteine residues in CysB (Cys1058/1061/1071/1076). For each sequence, secondary structure predictions $\left(2^{\circ}\right)$ are shown for the REPROFsec (upper line) and PROFsec (lower line) algorithms of the PredictProtein server (red bars, $\alpha$-helix; blue bars, $\beta$-strand); solvent accessibility (acc) is indicated by yellow (buried) or blue (exposed) bars; regions of significant difference between wild-type and variant sequences are boxed by broken red lines in the Ile1070Asn panel.

feature or if it seems specific to patients carrying mutations in the protein's ZNF2 domain.

In this study we also reported a novel MDPL patient carrying the recurrent in frame deletion p.S605del. In fact, this syndrome was associated in 2013 for the first time to the POLD1 gene by some of us, where the in frame deletion (c.1812_1814del, p.S605del) was reported in four unrelated MDPL patients [11]. This study was followed by two recent reports where a total of nine patients with the same deletion were described $[17,18]$. This is likely a mutation hotspot leading to MDPL syndrome.

As previously described, the p.Ser605del mutation is located in the polymerase active domain of POLD1, while the p.Arg507Cys missense mutation is located in the C-terminal part of the exonuclease domain. Functional studies have shown that the p.Ser605del results in an enzyme which is inactive as a polymerase but retains exonuclease activity [11], while the p.Arg507Cys is adjacent to a variant that has been described in a cell line of human colorectal cancer, which slightly reduces the fidelity of DNA replication [27]. Unlike these mutations, based on previous in vivo and in vitro studies as well as on novel in silico predictions described in this work, it is expected that all catalytic functions of POLD1 are affected by the p.Ile1070Asn missense mutation, and this may well result in novel and more severe clinical features. Therefore, we have re-evaluated the clinical description of patient 1 after the identification of the causative mutation. It is obvious that various clinical features of our patient overlap with those of the other MDPL patients. As shown in Table 1, patient 1 developed lipodystrophy, progeroid facial features, muscle wasting and dental crowding which were among the most prominent clinical characteristics of the previously reported patients. Normal hearing was also reported in 3 patients with MDPL syndrome by Lessel et al., indicating that this clinical feature may not always be associated with the syndrome. Our patient displayed severe postnatal growth failure, leading to very severely reduced linear growth with a Z-score of -6.6 at 24 years of age. Of note, Lessel et al. also reported 7 patients with short stature, but these showed milder growth retardation (range -0.9 to -3.7 SDS) (Table 1) while the stature of the other patients carrying POLD1 mutations reported by Pelosini et al. and Reinier et al. were closer or into normal Z-score ranges (please see Table 1). On the other hand, our patient showed hypogonadism, which has been reported in several male patients carrying POLD1 mutations but also in a female patient by Reinier et al. and in one of the patients carrying the p.E1067K mutation in the ZNF2 domain by Ajluni et al. As shown in Table 1 glucose intolerance test indicated a prediabetic status in our patient 1 . Unfortunately, she passed away before further biochemical measurements could be performed to confirm her diabetic status. As well, dyslipidemia, which is an important trait related to this syndrome, could not be searched for. Moreover, some other noteworthy differences have been noted. Indeed, it is important to note that patient 1 showed a much more severe phenotype than all MDPL patients described previously carrying the p.Ser605del or p.Arg507Cys mutation, namely featuring more striking progeroid features and premature death. Furthermore, this patient showed a high susceptibility to severe respiratory tract infections, as well as arachnodactyly and bulbar dysphagia which were never described in MDPL patients before. As well, the two related patients 
reported by Ajluni et al. carrying a new mutation in the ZNF2 domain of POLD1 did not show the same severe clinical features observed in patient 1 , and showed only some of the previously reported signs of MDPL patients (Table 1). Indeed, the patients reported by Ajluni et al. did not present with generalized lipodystrophy, progeroid features or short stature as our patient, nor severe insulin resistance or deafness as MDPL patients, nonetheless, they shared some of our patient's and previous MDPL signs as small mandibles, paucity of fat in the extremities and joint stiffness [20]. Overall, phenotypic comparison between patients carrying POLD1 mutations shows a clear phenotypic variability. This observation is also true for instance for patients carrying LMNA gene mutations, in whom not only different mutations cans cause totally different syndromes, but different missense changes of the same aminoacid can cause different phenotypic expressivity or different disorders [28]. Indeed, the aminoacid sequence is known to affect the secondary structure of the proteins, thus possibly changing the interaction capabilities of the mutated protein with tissue-specific partners. Furthermore, differential allelic expression has been suggested as a potential mechanism for the phenotypic variability in laminopathies [28] and it could be interesting to test the existence of physiologically low and high expressing alleles in the POLD1 locus (upon SNP patterns), and the consequences of the different mutations observed in MDPL patients on POLD1 expression levels. Although functional and molecular investigations would help to confirm the involvement of the novel mutation reported in the patient's clinical phenotype, the variant p.Ile1070Asn was predicted as pathogenic through the different in silico prediction databases and deep bioinformatics analyses did not reveal another pathogenic variant that could modify the severity of the clinical phenotype, considering that $88 \%$ of the coding regions of the genome were covered at $20 \mathrm{X}$. However, the presence of a modifier variant in the non-covered regions cannot be ruled out.

Last, but not least, mutations of POLD1 affecting the exonuclease domain have been described in colorectal cancer and/or polyposis predisposition [21]. It has been suggested that mutations in the exonuclease domain may contribute to the high mutation rate observed in tumors while mutations in the polymerase domain would be responsible for the appearance of other clinical features including lipodystrophy [14]. The recent study from Lessel and colleagues also highlighted the differences in the localization of "progeroid" and "malignant" types of substitutions in the POLD1 protein structure using molecular modeling data; these provide evidence of likely different mechanisms related to progeroid and cancerassociated POLD1 mutations [18]. Further functional studies will be needed to clarify the genotype-phenotype relationships in patients with rare deleterious germline mutations in the POLD1 gene, namely depending on the impacted domain of the protein and its corresponding function.

In conclusion, our study shows that patients carrying POLD1 mutations add to the wide phenotypic spectrum of premature aging syndromes, notably with particularly severe phenotypes. We thus report two novel cases affected with the rare MDPL syndrome and have identified a novel likely pathogenic variant in the ZNF2 domain of POLD1, which adds further evidence for the role of this gene in causing MDPL syndrome. Adding to previous reports, this work underlines the need to include this gene among the candidate genes tested in patients affected with atypical and/or severe progeroid syndromes. At this stage, since different POLD1 mutations can lead to major phenotypic variability, further functional work is needed to unravel the pathophysiological mechanisms underlying MDPL syndrome. Namely, it will be interesting to see whether mutations in different POLD1 domains can lead to nuclear disorganization, which may represent a major pathophysiological link with LMNA mutation-associated lipodystrophies. These explorations will be also essential to establish new targets for the discovery of appropriate therapeutic strategies.

\section{REFEREN C E S}

[1] Shastry S, Simha V, Godbole K, Sbraccia P, Melancon S, Yajnik $\mathrm{CS}$, et al. A novel syndrome of mandibular hypoplasia, deafness, and progeroid features associated with lipodystrophy, undescended testes, and male hypogonadism. J Clin Endocrinol Metab 2010;95:E192-7.

[2] De Sandre-Giovannoli A, Bernard R, Cau P, Navarro C, Amiel J, Boccaccio I, et al. Lamin a truncation in Hutchinson-Gilford progeria. Science 2003;300:2055. 
[3] Eriksson M, Brown WT, Gordon LB, Glynn MW, Singer J, Scott L, et al. Recurrent de novo point mutations in lamin a cause Hutchinson-Gilford progeria syndrome. Nature 2003;423:293-8.

[4] Puente XS, Quesada V, Osorio FG, Cabanillas R, Cadinanos J, Fraile JM, et al. Exome sequencing and functional analysis identifies BANF1 mutation as the cause of a hereditary progeroid syndrome. Am J Hum Genet 2011;88:650-6.

[5] Yu CE, Oshima J, Fu YH, Wijsman EM, Hisama F, Alisch R, et al. Positional cloning of the Werner's syndrome gene. Science 1996;272:258-62.

[6] Navarro CL, De Sandre-Giovannoli A, Bernard R, Boccaccio I, Boyer A, Genevieve D, et al. Lamin a and ZMPSTE24 (FACE-1) defects cause nuclear disorganization and identify restrictive dermopathy as a lethal neonatal laminopathy. Hum Mol Genet 2004;13:2493-503.

[7] Navarro CL, Cadinanos J, De Sandre-Giovannoli A, Bernard R, Courrier S, Boccaccio I, et al. Loss of ZMPSTE24 (FACE-1) causes autosomal recessive restrictive dermopathy and accumulation of Lamin a precursors. Hum Mol Genet 2005;14: 1503-13.

[8] Henning KA, Li L, Iyer N, McDaniel LD, Reagan MS, Legerski R, et al. The Cockayne syndrome group a gene encodes a WD repeat protein that interacts with CSB protein and a subunit of RNA polymerase II TFIIH. Cell 1995;82:555-64.

[9] Mallery DL, Tanganelli B, Colella S, Steingrimsdottir H, van Gool AJ, Troelstra C, et al. Molecular analysis of mutations in the CSB (ERCC6) gene in patients with Cockayne syndrome. Am J Hum Genet 1998;62:77-85.

[10] Palles C, Cazier JB, Howarth KM, Domingo E, Jones AM, Broderick $\mathrm{P}$, et al. Germline mutations affecting the proofreading domains of POLE and POLD1 predispose to colorectal adenomas and carcinomas. Nat Genet 2013;45: 136-44.

[11] Weedon MN, Ellard S, Prindle MJ, Caswell R, Lango Allen H, Oram R, et al. An in-frame deletion at the polymerase active site of POLD1 causes a multisystem disorder with lipodystrophy. Nat Genet 2013;45:947-50.

[12] Mossi R, Ferrari E, Hubscher U. DNA ligase I selectively affects DNA synthesis by DNA polymerases delta and epsilon suggesting differential functions in DNA replication and repair. J Biol Chem 1998;273:14322-30.

[13] Waga S, Stillman B. The DNA replication fork in eukaryotic cells. Annu Rev Biochem 1998;67:721-51.

[14] Nicolas E, Golemis EA, Arora S. POLD1: central mediator of DNA replication and repair, and implication in cancer and other pathologies. Gene 2016;590:128-41.

[15] Prindle MJ, Loeb LA. DNA polymerase delta in DNA replication and genome maintenance. Environ Mol Mutagen 2012;53:666-82.
[16] Sanchez Garcia J, Ciufo LF, Yang X, Kearsey SE, MacNeill SA. The C-terminal zinc finger of the catalytic subunit of DNA polymerase delta is responsible for direct interaction with the B-subunit. Nucleic Acids Res 2004;32:3005-16.

[17] Reinier F, Zoledziewska M, Hanna D, Smith JD, Valentini M, Zara I, et al. Mandibular hypoplasia, deafness, progeroid features and lipodystrophy (MDPL) syndrome in the context of inherited lipodystrophies. Metab Clin Exp 2015;64:1530-40.

[18] Lessel D, Hisama FM, Szakszon K, Saha B, Sanjuanelo AB, Salbert BA, et al. POLD1 germline mutations in patients initially diagnosed with Werner syndrome. Hum Mutat 2015;36:1070-9.

[19] Pelosini C, Martinelli S, Ceccarini G, Magno S, Barone I, Basolo $A$, et al. Identification of a novel mutation in the polymerase delta 1 (POLD1) gene in a lipodystrophic patient affected by mandibular hypoplasia, deafness, progeroid features (MDPL) syndrome. Metab Clin Exp 2014;63:1385-9.

[20] Ajluni N, Meral R, Neidert AH, Brady GF, Buras E, McKenna B, et al. Spectrum of disease associated with partial lipodystrophy (PL): lessons from a trial cohort. Clin Endocrinol (Oxf) 2017.

[21] Bellido F, Pineda M, Aiza G, Valdes-Mas R, Navarro M, Puente DA, et al. POLE and POLD1 mutations in 529 kindred with familial colorectal cancer and/or polyposis: review of reported cases and recommendations for genetic testing and surveillance. Genet Med 2016;18:325-32.

[22] Li H, Durbin R. Fast and accurate short read alignment with burrows-wheeler transform. Bioinformatics 2009;25:1754-60.

[23] McKenna A, Hanna M, Banks E, Sivachenko A, Cibulskis K, Kernytsky A, et al. The genome analysis toolkit: a MapReduce framework for analyzing next-generation DNA sequencing data. Genome Res 2010;20:1297-303.

[24] Lek M, Karczewski KJ, Minikel EV, Samocha KE, Banks E, Fennell T, et al. Analysis of protein-coding genetic variation in 60,706 humans. Nature 2016;536:285-91.

[25] Netz DJ, Stith CM, Stumpfig M, Kopf G, Vogel D, Genau HM, et al. Eukaryotic DNA polymerases require an iron-sulfur cluster for the formation of active complexes. Nat Chem Biol 2012;8:125-32.

[26] Yachdav G, Kloppmann E, Kajan L, Hecht M, Goldberg T, Hamp T, et al. PredictProtein-an open resource for online prediction of protein structural and functional features. Nucleic Acids Res 2014;42:W337-43.

[27] Yoshida R, Miyashita K, Inoue M, Shimamoto A, Yan Z, Egashira A, et al. Concurrent genetic alterations in DNA polymerase proofreading and mismatch repair in human colorectal cancer. Eur J Hum Genet 2011;19:320-5.

[28] Rodriguez S, Eriksson M. Low and high expressing alleles of the LMNA gene: implications for laminopathy disease development. PLoS One 2011;6:e25472. 